\title{
La ciudad en cuatro poetas en lenguas indígenas mexicanas
}

The City in four Poets in indigenous Languages

\author{
Krishna NARANJo Zavala \\ ORCID: 0000-0001-9068-8539 \\ Universidad de Colima, México \\ krish@ucol.mx
}

Resumen:

El artículo tiene como propósito explorar la percepción de la ciudad desde la mirada de cuatro poetas que conforman el espectro de la poesía indígena mexicana contemporánea: Karen Peñate (Tumbalá, Chiapas), Mikeas Sánchez (Chapultenango, Chiapas), Martín Tonalméyotl (Chilapa de Álvarez, Guerrero) y Wildernain Villegas (Mérida, Yucatán). El análisis inicia el recorrido - a propósito de la presencia de la ciudad - desde lo mítico hasta algunos momentos clave de la literatura mexicana del siglo Xx para indagar, finalmente, en la configuración de la ciudad en los trabajos de cuatro escritores.

Este detenimiento en cada poema promueve la discusión en torno a la noción dinámica y fluctuante de "literatura indígena", así como a los tópicos atribuidos, por prejuicio o inercia, a la producción poética en lenguas originarias, como la naturaleza o el paisajismo lírico, pero me detengo en la representación de la ciudad que se convierte en un espacio sobre el que se ejerce una mirada crítica, incluso en intersección con el espacio comunitario. De igual manera, se integran los testimonios de los escritores sobre la presencia de la ciudad como catalizador de escritura. Estos valiosos aportes posibilitan la reflexión en torno al vínculo entre el autor y el poema, para tal ejercicio me 
apoyo en Helena Beristáin; de igual manera, en Luz María Lepe Lira, en cuanto a los tipos de literatura indígena y las posturas de resistencia.

Palabras clave:

literatura mexicana, poesía indígena, ciudad, comunidad, testimonio.

\section{Abstract:}

The purpose of the essay is to explore the perception of the city from the perspective of four poets that shape the spectrum of contemporary Mexican indigenous poetry: Karen Peñate (Tumbalá, Chiapas), Mikeas Sánchez (Chapultenango, Chiapas), Martín Tonalméyotl (Chilapa de Álvarez Guerrero) and Wildernain Villegas (Mérida, Yucatán). The analysis begins the journey — about the presence of the city - from the mythical to some key moments of 20th century Mexican literature to finally inquire into the configuration of the city in the works of these four writers; pausing in each poem leads us to expand the discussion around the dynamic, problematic and fluctuating notion of "indigenous literature", as well as the topics attributed, by prejudice or inertia, to the poetic production in native languages, such as nature or lyrical landscaping. However, I stop at the representation of the city that becomes a space on which a critical gaze is exercised, even in intersection with the community space. Similarly, writer's testimonies of the city's presence as a catalyst for writing are integrated. This valuable contribution from each poet will allow reflection on the close interaction between the author and the poem, for this exercise I rely on Helena Beristáin. Likewise, on Luz María Lepe Lira regarding the types of indigenous literature and the postures of resistance.

Keywords:

Mexican literature, indigenous poetry, city, community, testimony.

DOI: https://doi.org/10.36798/critlit.v0i23.387 
Recibido: 24 de marzo de 2021

Aceptado: 17 de mayo de 2021

El objetivo de este trabajo es ahondar en la experiencia de la ciudad en cuatro poetas que forman parte de la literatura indígena contemporánea. Además del español, escriben respectivamente en ch'ol, zoque, maya y náhuatl. Se indaga, entre los propios autores, el tratamiento de la ciudad como tema y/o catalizador de escritura, considerando que, por lo general, existe un horizonte de expectativas en torno a la producción literaria en lenguas indígenas; por ejemplo, se le atribuye invariablemente la presencia de la naturaleza. Debo reconocer que mi lectura es de alguna manera parcial en tanto que mi análisis surge desde el español. Por tal razón, es imprescindible el diálogo con cada uno de ellos. Formulé las siguientes interrogantes que variaron en función del trabajo de cada poeta, pero en términos generales se encaminaron hacia dos puntos: 1) la ciudad y la escritura; 2) la ciudad como espacio de denuncia: ¿consideras que la ciudad detona material para tu escritura poética?, ¿cómo influye en tu proceso creativo?, ¿concibes la ciudad, desde tu oficio poético, como un espacio político para denunciar o manifestar crisis sociales de diversa índole?, ¿consideras que en tu poesía existe una suerte de antítesis entre un escenario citadino y uno rural?

El artículo se organiza en tres apartados, en el primero hago hincapié en discusiones ineludibles sobre la etiqueta "indígena", asimismo en la relación autor-contexto-obra, también en las literaturas latinoamericanas que se ubican al margen del canon así como en los rasgos compartidos en la poesía mexicana en lenguas originarias; en esta sección figuran los aportes de Genara Pulido. En el siguiente, realizo un recorrido sin un orden bien definido que recuerda la importancia de los mitos fundacionales prehispánicos para el imaginario mesoamericano e incluso contemporáneo con respecto a la conformación de la nación mexicana. También menciono, de manera transitoria, la idealización de la ciudad en la literatura novohispana, su presencia en otros momentos de las letras de nuestro país como en la vanguardia mexicana del siglo pasado 
para, finalmente, explorar la ciudad desde cuatro poetas indígenas: Juana Karen Peñate, Mikeas Sánchez, Wildernain Villegas y Martín Tonalméyotl. Me apoyo en consideraciones de Helena Beristáin a fin de discernir en el yo, tanto lírico como autoral, que resulta significativo en cada poeta; del mismo modo, en Luz María Lepe Lira, para comprender desde una mirada más amplia los tipos de literatura indígena, además de la colonialidad y decolonialidad como fenómenos implicados en los registros de la urbe.

\section{Voces poéticas en lenguas indígenas}

A la literatura mexicana contemporánea escrita en lenguas originarias, además del español, se le ha nombrado “indígena”, etiqueta que promueve diversas posturas: por un lado, de escritores que asumen su producción literaria inscrita en este ámbito; por el otro, de quienes cuestionan seriamente tal clasificación. Es imposible pasar por alto la discusión que, de primer orden, se vincula con la literatura escrita en lenguas indígenas. Desde mi punto de vista, para los estudios literarios es necesaria una luz que permita iluminar los rumbos de abordaje hacia dicha producción. Máxime, considerando que los estudios culturales han ganado terreno en el estudio y la discusión de la literatura situada al margen del canon. Estas clasificaciones no solo aplican a la producción referida, también en aquellas escrituras cuyas coordenadas geográficas, culturales, lingüísticas, políticas, estéticas, resultan esclarecedoras si es que interesa la intersección de estos elementos para aproximarse a ciertas obras literarias.

De acuerdo con Genara Pulido, para el reconocimiento amplio de la literatura latinoamericana fue necesario contemplar ángulos que, al menos desde principios del siglo XX no se consideraban; hacia finales de ese siglo e inicios del siguiente, los estudios literarios latinoamericanos motivaron en Norteamérica, Europa Occidental y algunas regiones de Latinoamérica, el interés por cuestionar el canon eurocéntrico que desestimaba a la literatura indígena, negra, popular, así como a la oralidad, una característica relevante de estas manifestaciones (Pulido 9). Tal cuestionamiento ha impactado 
positivamente porque estas literaturas poseen un sitio cada vez más visible en el panorama literario y crítico. La reflexión que planteó José Joaquín Blanco, que resulta vigente, sintetiza una visión necesaria en torno a la lírica indígena: "La poesía indígena, en todo caso, y no sólo a la antigua sino la que se sigue haciendo en nuestros días, merece capítulos propios y autosuficientes en las letras nacionales, y no una mera referencia a su traducción castellana, que siempre suena más a la tradición hispánica que a la indígena” (119).

Ahora bien, colocando el foco de atención en las voces poéticas mexicanas en lenguas indígenas, propongo repasar una serie de convergencias. En este trabajo menciono tres: 1) la presencia comunitaria como un eje que articula los discursos y representaciones líricas; 2) las genealogías literarias en tanto referentes de visiones de mundo que influyen, desde luego, en el terreno discursivo. Por ejemplo, algunos escritores mayas recuperan elementos cosmogónicos o referencias a luchas sociales, temas contenidos en El Popol $V$ uh, Rabinal Achí, o en el Ritual de los Bacabes, en conjunción con sus propuestas poéticas, conformando una suerte de entretejido textual. Mercedes de la Garza (2012) identifica los contenidos de estos libros: mítico, profético, ritual, médico, astronómico, calendárico, información histórica y legendaria, los cuales persisten en las voces poéticas actuales; por último, 3) la denuncia y movilización social en consonancia con los reclamos de los pueblos indígenas frente a las invasiones paramilitares, desapariciones forzadas, la degradación ambiental provocada por concesiones mineras e hidroeléctricas, entre otros temas — en este tenor encontramos la poesía de Ruperta Bautista, Irma Pineda o Hubert Matiúwaà-.

Lo anterior dibuja, grosso modo, la panorámica de la poesía mexicana en lenguas indígenas; es un amplio espectro temático en virtud de las culturas, escrituras y lenguas de las que participan sus autores. Cada vez surgen, en diversos puntos del país, propuestas literarias escritas en lenguas indígenas y en español. Asimismo, continúan escribiendo quienes cuentan con una trayectoria literaria desde hace varias décadas, como los autores en lengua maya, Jorge Miguel Cocom Pech, Briceida Cuevas Cob y Feliciano Sánchez Chan; en lengua náhuatl, Natalio Hernández; y, en zapoteco, Javier Castellanos, 
por mencionar algunos ejemplos. En las convergencias mencionadas es claro que la literatura brota de su dimensión social bien para expresarla, replantearla o subvertirla.

La Unesco proclamó 2019 como el Año Internacional de las Lenguas Indígenas a fin de que las discusiones giraran en torno a estas, a fortalecer su presencia y dar cuenta de la gravedad respecto a su extinción en todo el mundo. En México ha existido un adverso escenario histórico porque los proyectos de nación no han considerado su diversidad cultural y lingüística, es así que la literatura actual escrita en lenguas originarias confronta dicho horizonte, y al mismo tiempo lanza el reto de conseguir no solo interesados sino lectores en estos idiomas. No ahondaré en el tema porque significaría caminar en otra dirección, pero considero elemental aproximarnos a ciertas claves que destacan en un rico panorama de voces poéticas en lenguas indígenas mexicanas. El abordaje de la naturaleza es quizá, el de mayor relieve; el tema va de la mano con una serie de saberes de diversos ámbitos. Si por un lado tenemos a la naturaleza que constituye un manantial vastísimo de auténticos conocimientos sobre el mundo y el ser humano en un ciclo vital, ¿qué se escribe de la ciudad?, ¿qué confluencias se atisban en poetas de distintas regiones a partir del tema urbano?

\section{Del mito a la ciudad: un mosaico literario}

Vamos a la ventana histórica: los códices prehispánicos son valiosas fuentes sobre el tema de la conformación de la nación mexicana que, a nivel mítico, simbólico e histórico, han dejado huella en el ideario de los mexicanos; como valiosos son también los textos que se produjeron durante la etapa colonial. León-Portilla, citando a Del Paso y Troncoso, refiere el mito con el vocablo náhuatl, tlamachiliztlabtoliazanilli, "relatos o relatos de la palabra del saber" (10), dejando claro el peso de la oralidad para los pueblos mesoamericanos. De igual manera, el historiador menciona: "Cierto es que muchas veces lo mítico y lo histórico entre los aztecas parecen confundirse para dar lugar al discurso legendario" (22). 
En la conformación del México-Tenochtitlan, como lo hacen ver los Códices Matritenses, tuvo que ver, además de la peregrinación, la importancia simbólica del hecho fundacional donde sobresalen estos elementos: yóllotl (corazón), tenochtli (tunal), cuaubtlequetzi (águila que se yergue). El corazón de Cópil debía sembrarse entre los cañaverales. De este nacería el tunal que se apoyaría en la piedra donde posaría el águila. Chimalpain dejó una serie de Relaciones Históricas — retomo aquí la observación de León-Portilla sobre el vínculo entre lo mítico y lo histórico- que deja apreciar un relato sobre la guerra entre los mexicas frente a los tepanecas, los texcaltepecas, los malinalcas y los de Tolula; esto inscrito en el año 10-Calli, 1281. ${ }^{1}$ Finalmente, dicho relato tendrá un sentido de triunfo mexica tras la hazaña de Tenochtli y Cuauhtlequetzqui. Al ver que, en efecto, el corazón extraído de Cópil floreció, dando lugar al tunal sobre la piedra donde se erguiría el águila, Cuauhtlequetzqui mencionó: "Esta será nuestra fama: en tanto que dure el mundo, así durará el renombre, la gloria, de México Tenochtitlan" (en León-Portilla 167). Hemos visto la delgada línea entre lo histórico y lo mítico; de hecho, el territorio de Aztlán se ha sometido al debate: "La polémica plantea hoy la disyuntiva entre una Aztlán mítica y una real" (López Austin y López Luján 210). México-Tenochtitlan, el imperio mexica, durará doscientos años. Durante el auge de su poderío llegan los conquistadores españoles:

Tras la victoria española sobre Mexico-Tenochtitlan, los europeos fundaron el centro del dominio colonial sobre las ruinas de la ciudad. Los conquistadores describieron las creencias, las costumbres y las instituciones de los vencidos tomando

1 Tenochtli, enterado de dicho propósito, comparte su pesar con Cuauhtlequetzqui, quien, enérgico, reitera que protegerá al territorio mexica en Chapultepec. Sucedió la guerra y Cuauhtlequetzqui atrapó a Cópil para extraerle el corazón y enseguida le ordenó a Tenochtli que lo sembrara entre los cañaverales. Luego de que germinó el corazón de Cópil, Tenochtli vio la escena fundacional: el águila devorando la serpiente (León-Portilla 163-167). 
como principal modelo a los mexicas. (López Austin y López Luján 210- 211)

De la época prehispánica fueron los mitos, relatos orales, códices, pinturas murales, entre otras fuentes, referencias importantes sobre la ciudad; en la Nueva España será especialmente la crónica. No obstante, daré un salto temporal para situarme en Bernardo de Balbuena, poeta del siglo XVI, quien escribió por encargo de Isabel de Tobar y Guzmán, una descripción de la Nueva España. Escrita en tercetos, Balbuena publica Grandeza mexicana, texto del que se reconoce su capacidad de versificación, pero se somete a opiniones encontradas. José Joaquín Blanco subraya la idealización de la ciudad; en sus palabras es un "idilio urbano" (216). Es la mirada poética la que elegirá los pasajes, las luces u oscuridades de la urbe y la dinámica social. Si en la etapa colonial se tendía al ensalzamiento, a la mirada arquitectónica en la descripción detallada de la ciudad, irá cobrando un sentido más complejo en cuanto a una mayor subjetividad de la vOz poética sobre la urbe. Incluso, en pleno siglo XX, llega a colocarse como tema principal en el movimiento Estridentista, movimiento de la vanguardia mexicana que incluyó a las artes plásticas.

El encantamiento que la ciudad moderna provocaba en los estridentistas, nos invita a pensar cómo la visión actual sobre la urbe tiende más bien a una asociación de aspectos negativos, al desencanto, pese a los capitales culturales que posee, por ejemplo, la ciudad de México, siguiendo a García Canclini. Aunque el antropólogo se refiere a la capital del país, me parece que es representativa de lo que ocurre en el territorio mexicano: descomposición social, crecimiento desmedido de las ciudades, problemas medioambientales, entre otros. Ello no impide que la Ciudad de México conserve su encanto, agridulce en ocasiones, como lo podemos ver en las crónicas de Juan Villoro en El vértigo horizontal, de reciente publicación. De acuerdo con Canclini: "Pero si la capital mexicana es hoy una ciudad más desordenada que barroca es porque los imaginarios en conflicto han trabajado más para destruirse o ignorarse que para erigir una utopía compartida" (9). 
Exploremos ahora el tratamiento de la ciudad en la poesía actual escrita en lenguas indígenas y en español. Considerando que se le adjudica el abordaje de la naturaleza como un "tema obligado" y reconociendo que es un tópico recurrente, resulta importante la observación de Luz María Lepe Lira sobre el hecho de que la literatura indígena abraza diversas preocupaciones temáticas y estéticas. Añado que un mismo autor puede incursionar en distintos estilos y temáticas, como ocurre con numerosos poetas mexicanos. Me sitúo en el ejemplo particular de la poeta Ruperta Bautista, poeta bilingüe (tsotsil-español), quien escribe con un tono denunciatorio sobre el capitalismo, la violencia ejercida contra los pueblos indígenas y, por otro lado, se interesa por el tiempo y el calendario maya, vetas que evocan una espirtualidad lírica. Lepe Lira menciona tres tipos de literatura indígena: la que se encauza a recuperar la memoria, otra que recrea la tradición y la híbrida, la cual me interesa subrayar. De acuerdo con la investigadora el hibridismo cultural se da en dos maneras:

la unión del pasado prehispánico con el mundo contemporáneo, y la mezcla de los sincretismos culturales de los mass media con los mestizajes coloniales. El primer tipo es una hibridación del contexto social y cultural del México contemporáneo en diálogo con el pasado prehispánico, esta mezcla de tiempo y espacio (sin contradicción para el pensamiento indígena, provoca sorpresa al lector occidental porque el tiempo lineal y progresivo de la modernidad, no concuerda con el tiempo cíclico del pensamiento indígena. (69)

La poesía contemporánea en lenguas indígenas coloca a la ciudad como un espacio en permanente conflicto, originado por el capitalismo, la desigualdad social o el narcotráfico. En numerosos poemas sobresale un tratamiento maniqueo (ciudad-comunidad, naturaleza) pero también interseccionado. De igual manera, los espacios son híbridos porque se entretejen dinámicas de vida vinculadas con la ciudad; otras, con el ámbito rural, incluso cuando el territorio representado en el poema es el comunitario, pero con la presencia del 
sello urbano, sobrevendrá alguna crítica, alguna mirada peyorativa sobre el entorno que se ha transformado.

Revisemos a cuatro poetas mexicanos nacidos entre los setenta y ochenta, quienes se destacan por sus trayectorias literarias y ámbitos afines como la educación bilingüe, la traducción, la gestión cultural, la docencia o la conducción de programas televisivos o radiofónicos. A partir de un corpus poético leeremos que la ciudad se manifiesta bien como espacio central o coyuntural. ${ }^{2}$ Estos abordajes se enuncian desde entornos específicos donde la identidad, la lengua, así como la participación en determinada comunidad son elementos que se tamizan en los textos. No es el objetivo del trabajo ahondar en la relación entre autor-texto; sin embargo, considero que la dimensión ficcional de la literatura, aunque es crucial asumirla en todo ejercicio creativo, se manifiesta con menor densidad en la producción lírica en lenguas indígenas. Podríamos decir, de acuerdo con Helena Beristáin, que esto ocurre, en términos generales, con el género lírico, pero asumimos que hoy en día la relación autor-texto, origina diversos puntos de vista. Al respecto, la académica se detiene en estas dos posturas, las cuales sintetiza con claridad:

Pedro Salinas, apoyándose en John Crowe Ransom, dice que el drama es un excelente símbolo de la poesía pues el poeta no habla en su nombre sino en el nombre de un personaje que se da por supuesto. El poeta, pues, se pone una máscara que es el lenguaje poético, y luego se endosa un disfraz que es el de la situación por él elegida. Por ello, dice Salinas, la poesía, más que una experiencia real, es una experiencia dramática. Al contrario de estos autores, nosotros pensamos que,

${ }^{2}$ Los y las poetas son: Juana Karen Peñate (1979, Tumbalá, Chiapas) con “Kña’ mayte’lum” / “Madre Selva”; Mikeas Sánchez (1980, Chapultenango, Chiapas) con el poema "Nereyda'is myabaxäyu nwyt New’York. / "Nereyda se soñó en New York"; Wildernain Villegas Carrillo (1981, Mérida, Yucatán) con "Junp'éel k’uj ku ch'inik u báat” / "Un dios lanza su hacha” y Martín Tonalmeyotl (Atzacoaloya, municipio de Chilapa de Álvarez, Guerrero, 1983), "Notlaltipaktsin" / "Mi tierra". 
precisamente, el poema lírico es el tipo de discurso literario en el cual el yo enunciador no desempeña un papel ficcional (a diferencia del narrador de la novela, el cuento, la epopeya, etcétera, y a diferencia del autor que construye un drama y del actor que lo representa), pues, aunque cumple un papel literario — dice Mignolo- éste no es un papel ficticio: se desarrolla fuera de la ficción. (49)

Estas dos consideraciones que Helena Beristáin coloca en el terreno del análisis poético nos invitan a reflexionar en torno a la poesía indígena contemporánea en relación con el tema de la ciudad. En mi opinión, las voces líricas que revisaremos se inclinan hacia la segunda postura, en tanto las referencias a los espacios corresponden, en algunos casos, a los espacios reales de los poetas. Ocurre lo mismo con los temas tratados: la violencia no es una mera representación sino un reflejo de la realidad social que forma parte del entorno de su autor; la potencia poética resulta efectiva para apuntar, denunciar o simplemente reflejar escenarios complejos del México actual; mencioné la violencia como ejemplo, pero se incluyen otros fenómenos sociales como la migración que implica la aculturación y, en gran medida, la pérdida de elementos culturales valiosos, la añoranza por el lugar de origen o por articular la lengua materna.

\section{La metrópoli, la tierra: cuatro poetas sobre la ciudad}

Comencemos con la poeta ch'ol, Juana Karen Peñate (1979, Tumbalá, Chiapas), ${ }^{3}$ con el poema "Kña' mayte’lum" / "Madre Selva",

${ }^{3}$ Juana Karen Peñate es originaria de Ejido Emiliano Zapata, Tumbalá, Chiapas. Es licenciada en Derecho por el Centro de Estudios Superiores de Tapachula, Chiapas. Cuenta con cursos y diplomados en creación literaria. Asimismo, se ha desempeñado en la traducción en su lengua ch'ol en el Centro Estatal de Lenguas, Arte y Literatura Indígena (CELALI), ha sido profesora bilingüe y coordinadora de proyectos culturales en el H. Ayuntamiento del Municipio de Tumbalá. También se ha desempeñado en la promoción y gestión 
del libro de poesía Corazón de selva / Ipusik'al matyelum (Pluralia Ediciones 2013). Cabe señalar que la escritora, recibió en 2020, un importante galardón por su libro de poesía Isoñil ja'al / Danza bajo la lluvia, se trata del Premio de Literaturas Indígenas de América (PLIA). En el poema mencionado se refleja una oposición entre ciudad-naturaleza. El poemario, desde el título, demuestra una clara elección poética: la selva como el sitio que la vio nacer y en donde entreteje su corporalidad; cuerpo y ambiente se corresponden. Sin embargo, frente a los manantiales, montañas o ríos que integran el universo lírico de Juana Karen Peñate, la referencia de la ciudad es un punto de quiebre. Leamos el poema mencionado donde el yo poético lanza una solicitud a la Madre Tierra, petición que emparenta al texto con la oración. El primer fragmento corresponde a la lengua ch'ol:

Mach komik sajtyel ilaj tyi kolem tyejlum,

Mach käyäyik chumtyäl che' maxtyo tyechbilik kcha'añ

[ñaxambä its 'ijbal jk'ay.

Majtyañ ak'eñoñ ili ak'lel ik'äk' al lak piälob,

Kambeñoñ kwuty tyi' yojlil kña' matye'lum.

(9)

No quiero perderme en la inmensidad de la metrópoli,

No desamparo mi habitar antes de iniciar la primera letra de mi canto.

Regálame esta noche la luz de la multitud, y despiértame en la profundidad de la Madre Selva.

de la cultura, así como en la conducción de un programa televisivo, Las voces de siempre. Impartió clases en la Universidad Intercultural de Chiapas, sede Yajalón. Obtuvo el tercer lugar en el Premio de Narrativa "Y el Bolom dice" y el Premio de Poesía Indígena Pat O’tan. En 2020 se hizo acreedora del PLIA por su libro de poesía Isoñil ja'al / Danza bajo la llwvia (Editorial Herder México y Tonalmeyotl, "Xochitlájtoli: Juana Karen"). 
A propósito del poema citado, Juana Karen Peñate confirmó la relevancia del lugar de origen que, en su caso, se vincula con la selva y se presenta como escenario y tema de escritura poética. Se observa un reconocimiento de ambos espacios, pero elige el primero que es el ambiente más ligado a la naturaleza como fuente de creación. En todo caso acude a la ciudad, como se lee en el fragmento del poema citado para reiterar lo significativo de la Madre Selva:

Si me olvido de mis raíces entonces de qué escribo, a quién le elevo mi canto, si solo hablara de la ciudad no tendría sentido, porque mi humanidad se mueve en los dos mundos, selva y ciudad y no hay vuelta atrás. Nací y crecí en la selva, viví y dormí en la ciudad, ahora trabajo en mi pueblo y viajo a la ciudad. Entonces mi caminar gira en estos dos mundos y por lo tanto no debo de olvidarme de ninguno de los dos. (Peñate, Entrevista oral)

Compartió la importancia de no desarraigarse del territorio que la vio nacer que, desde luego, guarda estrecha relación con su poesía:

Siempre he tenido presente que selva y ciudad son dos espacios esenciales que han sido como parte del proceso de mi caminar, no solo para mi trabajo poético sino en mi formación profesional, en lo laboral y obviamente en mi trabajo creativo . . en el año de 1997 salí del pueblo con destino a San Cristóbal, ella [mi madre] me dijo que tal vez no regresaría a mi pueblo a vivir, que tal vez me quedaría en la ciudad para hacer mi vida, ya que algunos y algunas que han salido de sus pueblos no regresan nunca, o si regresan, regresan cambiados en sus ideologías, o que ya se les olvidó el idioma, ya cambiaron su forma de comer, incluso desconocen a sus propios padres, y en ese tiempo aún con 18 años de edad, le aseguré a mi madre que regresaría a mi pueblo pronto .... El tiempo que viví en la Ciudad de San Cristóbal de las Casas, siempre tuve presente que no me podía olvidar de mis raíces y disfrutaba convivir con otros grupos de pueblos originarios 
y que a través de ellos me reconocía como parte de un pueblo con identidad. (Peñate, Entrevista oral)

Continuemos con el poema de Mikeas Sánchez (1980, Chapultenango, Chiapas) ${ }^{4}$; en una poética de tono narrativo, la voz lírica enuncia cómo una mujer joven se ve despojada de su matriz cultural zoque, del imperio Tzitzun —así se nombra en el texto-. La necesidad de migrar le ha llevado a enfrentar adversidades, no obstante, en medio de este desarraigo se da una suerte de ensoñación descrita en segunda persona por la voz lírica. Es así cómo el poema inicia con una refracción de Nereyda, una ore'yomo migrante (el significado de ore'yomo es "mujer zoque" de acuerdo con una nota al pie del libro de poesía Mojk'jäkä / Mokaya) quien visualiza, quizá de manera idílica, a la ciudad de Nueva York a través de una de las grandes tiendas departamentales estadounidenses. Leamos los siguientes fragmentos, el primero aparece en zoque. Este idioma es uno de los mas antiguos, porque, según Terje y Aguilar (2017) proviene de la civilización olmeca; existen evidencias arqueológicas y epigráficas que "permiten asegurar que los olmecas debieron de hablar protozoqueano o protomixe-zoqueano, es decir, un antepasado directo de las lenguas zoques actuales" (20). No deja de resultar asombroso y digno de reconocimiento que, pese a los golpes históricos que han recibido las lenguas indígenas mexicanas de parte del Estado, hoy se articulan y forjan caminos literarios - en los cuales aparece la urbe como catalizador de experiencias, sentimientos y reflexiones, usualmente de manera contrastante o

${ }^{4}$ Mikeas Sánchez es originaria de Ajway, Chiapas, México. Además de ser escritora, es productora de radio, traductora, docente y activista sobre el territorio zoque. Es una poeta prolífica. Ha publicado los libros de poesía: Maka mujsi Tumä jama / Y sabrás un día (2006), Äj' ngujkomo / Desde mi médula (2011), Mumure' tä' yäjktambä / Todos somos cimarrones (2012), Kobikiajubä jaye' / Selección poética (2012), Mokaya / Mojk'jäyä (2013) y Jujtzye tä wäpä tramapänh'ajä / Cómo ser un buen salvaje (2019). Sus poemas se incluyen en antologías de circulación nacional e internacional. Obtuvo el primer Premio de Narrativa "Y el Bolóm dice" y el Premio de Poesía Indígena Pat O’ tan. 
en interesante diálogo con la vida comunitaria referida por los y las poetas en lenguas indígenas-; dicho espectro plurilingüístico conforma, realmente, a la literatura mexicana.

Nereyda'is myabaxäyu nwwyt New’York

ne' yamumä' kiene tumä tuku'ma'aomo ñoyibäis Macy's tumäore'yomo

tumä pabiñomo pänajubä’ dä’ najsomo'ram

tumä' nkiae ne' pyoyubä koxtaksi'

ne' chajkienbäu'bäis dyagbajk'ajku'y

Yanu'kuis myuja'ajkujxye'

jaya' iri’ nijuräbä kubguyis nasakobajk'omo

Nereyda se soñó en New York

contemplando su reflejo en un escaparate de Macy's

una ore'yomo migrante

una muchacha nacida en el imperio Tzitzun

una niña huyendo descalza

lo más lejos posible de la orfandad

Jamás la grandeza de su linaje

habrá de compararse con ningún otro reino

Se aprecia una especie de antítesis a partir del territorio. Por un lado, vemos a la ciudad hegemónica, símbolo del capitalismo y, por el otro, el imperio Tzitzun. El Tzitzun es clave en la historia del pueblo zoque en tanto se trata del volcán Chichonal que, de acuerdo con Marina Alonso (131) hizo erupción el 28 de marzo de 1982, afectando la vida de los zoques, especialmente las localidades de varios municipios de Chiapas como Chapultenango, Ostuacán, Ocotepec y Francisco León. En el poema se reivindica la pertenencia al universo zoque, hecho que contrarresta la presencia de New York o de la tienda Macy's: “Jamás la grandeza de su linaje / habrá de compararse con la de ningún otro reino / pero creció con hambre 
/ y sus manos agrietadas por el frío / conocieron mejor el campo que su propio cuerpo" (47). La voz lírica enuncia la espinosa experiencia de una mujer migrante como Nereyda, por lo que el poema se antoja testimonial. Los poemas de Juana Karen Peñate y Mikeas Sánchez poseen como un rasgo similar la evocación a entidades femeninas relacionadas con la naturaleza: Madre Selva y Nasakobajk; el término zoque refiere a la Madre Tierra.

Los textos, además, lanzan peticiones de protección frente a lo que representa la urbe; en el caso del poema que ahora nos ocupa, se dirige a Nasakobajk con el fin de que brinde una protección espiritual a Nereyda, al tiempo que en la súplica — la anáfora es característica del tipo poema-oración - recupera un profundo reclamo de los pueblos indígenas a partir de la colonización: “Oh Nasakobajk si puedes escucharla / acércate a recoger su alma / acércate a saciar su sed de 500 años / acércate a rescatar su cuerpo injuriado / acércate a convertirla de nuevo en niña / aquella que jugaba con los guijarros / que circundan el cráter del Tzitzun" (47). Se trata de un poema que, en español, puede percibirse a manera de registro lírico cuyo tono es coloquial, pero al mismo tiempo la voz es contundente, poéticamente desgarradora.

Ahora bien ¿cómo se representa la ciudad en el poema de Wildernain Villegas Carrillo (1981, Mérida, Yucatán)? ${ }^{5}$ La estructura circular del poema “Junp'éel k’uj ku ch'inik u báat" / "Un dios lanza su hacha" favorece la clara expresión del planteamiento: la urbe es el ambiente propicio para el caos, incluso, un dios apuntala en la ciudad. El primer fragmento corresponde al maya:

${ }^{5}$ Wildernain Villegas es profesor-investigador en la Universidad Intercultural Maya de Quintana Roo. Escritor, traductor y académico. Es licenciado en Educación Secundaria con especialidad en Telesecundaria, egresado del Centro de Actualización del Magisterio Chetumal, Quintana Roo. En 2008 obtuvo el Premio Nezahualcóyotl de Literatura en Lenguas Mexicanas y en 2014 el Premio Internacional de Poesía del Mundo Maya Waldemar Noh Tzec. En 2011 fue campeón latinoamericano de oratoria en lenguas maternas Gran Señorío de Xaltocan, con la distinción de la medalla Madre Tierra. 
Junp'éel k'uj ku ch'inik u báat,

u nu'ukulo’ob sáasil ku kímilo’ob,

juulboon ku p'atik boox u juul,

chokopol xóobilo'ob ku ok'ochi'ibalo'ob,

luuk'e' ku wiits'il ti' k'áak' tsíimino’ob, ku je'elsiko’ob, ku buliko'ob. ${ }^{6}$

Un dios lanza su hacha:

mueren faroles,

El semáforo cambia a negro,

aúllan enloquecidos cláxones ,

el lodo salpica coches, los detiene,

los ahoga.

En otra parte,

alguna aldea se levanta de la sed,

el agua florece su rostro,

sacia chultunes

sartenejas,

\author{
cántaros, \\ sonrisas.
}

La floresta fecunda metáforas:

el aire es gigante que corre entre la selva,

la noche venado lamiendo su linaje, fulgores habitan la piel del ocelote, el rugido del tigre al rayo despierta, los sapos son pedazos de jade musical, goterones golpean el tambor de la tortuga.

${ }^{6}$ La semblanza del poeta y el poema citado se obtuvieron de: Tonalmeyotl, Martín, coordinador. "Xochitlájtoli: Wildernain Villegas Carrillo". Círculo de poesía. Revista electrónica de literatura, circulodepoesia.com/2019/01/xochitlajtoliwildernain-villegas-carrillo/ 
En cambio aquí,

la lluvia arruina discursos oficiales,

y esta bendita ciudad

se vuelve charca.

Si en la ciudad prevalece el bullicio representado en la oscuridad y en los automóviles, en el escenario opuesto afloran manifestaciones de armonía. Ha de advertirse un recurso estilístico empleado con afluencia en las literaturas indígenas: la personificación. Aunque por lo común se dirija hacia elementos de la naturaleza, en el poema de Wildernain Villegas lo apreciamos en objetos del turbio escenario citadino que plantea. Aparece una red de elementos de la naturaleza que permiten el flujo vital: "En otra parte, / alguna aldea se levanta de la sed, / el agua florece su rostro, / sacia chultunes, / sartenejas, / cántaros, / sonrisas". La comparación entre ciudad-naturaleza reside en que la primera significa estancamiento; en la segunda, los fenómenos como los rayos o la lluvia son actos creativos. La naturaleza engrendra poesía: "La floresta fecunda metáforas: / el aire es gigante que corre entre la selva, / la noche venado lamiendo su linaje, / fulgores habitan la piel del ocelote, / el rugido del tigre al rayo despierta, / los sapos son pedazos de jade musical ...”.

Un mismo elemento, así lo sugiere el poema de Villegas, adquiere connotaciones contrarias en función del entorno donde se desarrolle. La lluvia, en el escenario citadino "arruina discursos oficiales / y esta bendita ciudad / se vuelve charca". La voz lírica no pretende dialogar con dos mundos, ciudad-naturaleza; mas bien los distingue; parece estar "del lado" del segundo y desde ahí ejerce su mirada crítica. Sabe que un fenómeno tan natural como la lluvia llega a ser un estorbo en el ámbito citadino. En este punto, el poeta nos hace ver una escena que, aunque ordinaria, posee múltiples sugerencias: los discursos oficiales arruinados por la lluvia. Cada verso del poema de Wildernain Villegas se condensa en el sentido y en la imagen, no hay palabra o frase que den la impresión de sobra. En cuanto a su experiencia como escritor frente al tema que nos interesa, el poeta compartió su testimonio cuando llegó a estudiar a la ciudad de Chetumal, Quintana Roo, a la edad de aproximadamente veinte años: 
Acostumbrado siempre durante mi adolescencia e infancia a la vida de comunidad, Al llegar a la ciudad me topé con un paisaje totalmente diferente a mi comunidad, por supuesto, y no era una ciudad muy grande; Chetumal, Quintana Roo es una ciudad pequeña. Sin embargo, esta escritura sobre la ciudad en este primer momento de mi trabajo poético en el libro Lluvia que la noche dicta / Áak'abe' ku ya'alik táan u k'áaxal ja'... En la ciudad me topé con situaciones que, en mi pueblo, en mi comunidad no existían, no había, no se me presentaban y eso me orilló a escribir de la ciudad. Es un hecho poético muy interesante porque a pesar de que estaba en la ciudad siempre me llegaban a la memoria paisajes y elementos de mi pueblo, de mi comunidad, de la selva, de la milpa. Más que nada, la posibilidad de comparar la ciudad con el campo desde un punto de vista también emocional: el impacto de la ciudad en mis emociones, el impacto de la ciudad en mis pensamientos y comparar eso con lo que había vivido en la comunidad, me dio un material muy interesante, muy importante para poder escribir mirando la ciudad, pero desde los ojos de quien ha vivido siempre en una comunidad. Este encuentro poético entre la ciudad y la comunidad dio como resultado esos poemas. (Villegas, Entrevista oral)

Continuemos con el poema de Martín Tonalmeyotl (Atzacoaloya, municipio de Chilapa de Álvarez, Guerrero, 1983)7, "Notlal-

${ }^{7}$ Martín Tonalmeyotl (Martín Jacinto Meza) es originario de Atzacoaloya, municipio de Chilapa de Álvarez, Guerrero, México. Es campesino, profesor de lengua náhuatl, narrador, poeta y articulista. Es licenciado en Literatura Hispanoamericana por la Universidad Autónoma de Guerrero, maestro en Lingüística Indoamericana por el Centro de Investigaciones y Estudios Superiores en Antropología Social (CIESAS). Asimismo, cursó el doctorado de Lengua y Literatura Hispanoamericana en la Benemérita Universidad Autónoma de Puebla (BUAP). Coordinó las antologías: Xochitlajtoli. Poesía contemporánea en lenguas originarias de México (2019) publicada por Círculo de poesía, Flor de siete pétalos: espina florida de siete poetas mexicanas (2019) e In xochitl in kuikatl: 24 poetas contemporáneos en 
tipaktsin" / "Mi tierra". El poema traslada las representaciones citadinas hacia la propia comunidad — aquella que enuncia la voz lírica- para denunciar el clima desesperanzador donde la libertad de expresión, la corrupción, entre otros males sociales, aquejan a la región.

\author{
Notlaltipaktsin \\ ajkayanxiktsintle kampa mojmostla notlatlalouaj motomej \\ iluitsintle uan yokojkoyonijkej ika nejmotilistle \\ ajkosamalotsin uan sa yespetlantok \\ kiyajtsintle uan noyolkokojtok \\ ajakatsintle uan yokechtsontejkej \\ (68)
}

Esta es mi tierra

ombligo de sombras y ruidos de moto

un cielo acribillado por el miedo

un arcoiris manchado de rojo

una lluvia color tristeza

un viento con el cuchillo oxidado

(69)

En su libro de poesía, Istitsin ueyeatsintle okseke xochitlajtoltiny / Uña Mar y otros poemas (2019) me parece fundamental considerar desde dónde se escribe en tanto el marco geográfico es también territorio discursivo en el universo lírico de Martín Tonalmeyotl. En este sentido, cobra peso lo señalado por Beristáin sobre el yo lírico que se funde con el yo del autor. La académica, a propósito del poema de Pessoa, "El poeta es un fingidor", sostiene: "la situación de emisión del mensaje lírico es idéntica a la situación vivencial del poeta por lo cual, éste, en realidad suele realizar el esfuerzo contrario, para

lengua nábuatl (2020). Es autor de los libros de poesía: Tlalkatsajtsilistle / Ritual de los olvidados (Jaguar Ediciones 2016), Nosentlalilxochitlajtol / Antología personal (AEMAC 2017) y Istitsin ueyeatsintle okseke xochitlajtoltiny / Uña Mar y otros poemas (2019) (Cf. Istitsin ueyeatsintle okseke xochitlajtoltiny). 
desdoblarse y observarse desde fuera . ..”(52). Esta aseveración de Beristáin se refleja en las respectivas voces poéticas que aquí se reúnen. Por otro lado, no podemos reducir la creación poética a una manifestación estética de las experiencias personales de su autor. Desde mi juicio, la escritura poética entrevera aspectos vivenciales y ficcionales; en algunos poemas o libros de poesía, se presentará con mayor fuerza lo uno o lo otro. En el caso de Martín Tonalmeyotl, los ambientes y contextos representados en Uña Mar y otros poemas remiten a los reales de los que da registro el autor. Cabe señalar que los primeros poemas de este libro recuperan las bellezas, sonidos, texturas y ciertas fragancias de la naturaleza: la alegría que produce el fuego, el nido de las golondrinas, el tiempo de siembra; la contemplación del ambiente, sus fenómenos, la vida familiar en el medio rural, la evocación al padre, el tiempo onírico, la concepción de la poesía y la reflexión sobre el oficio: "Escribo para relatar los dolores de mis entrañas / porque de no hacerlo / nadie mas sabrá que me carcome" (23), por mencionar algunas preocupaciones. Los poemas van dirigiéndose hacia escenarios agridulces: la belleza se mezcla con la muerte o la tristeza.

La presencia de la tierra, la identidad, y un reclamo constante no a la urbe sino al lugar propio, son claves del poemario que nos llevarán a relacionar lo representado líricamente con el contexto del autor. A menudo se emplea la ironía, la descripción detallada de la atmósfera, así como de algunos acontecimientos y personas. Los versos de Istitsin ueyeatsintle okseke xochitlajtoltiny logran desautomatizar el lenguaje en el sentido de que aparecen escenas, asociaciones inusitadas que conforman una estética particular. El decir lírico va de la evocación, el conflicto interno pero, sobre todo, al testimonio de un pueblo atormentado, del que si bien se pondera su belleza, no se idealiza en tanto las complejidades sociales que enfrenta. Se retratan, asimismo, procesos de transculturación y la pérdida de la propia cultura. Leamos un fragmento de "Rostro de otros":

Aquí en nuestra propia tierra donde nuestros pies han marcado esperanza donde nuestras manos se han manchado de miseria 
donde nuestras flores lloran sangre

donde los nuestros quieren ser blancos

donde a los niños se les enseña a pisotear su idioma donde nuestros hermanos han desenterrado su ombligo para no sentir el sudor campesino donde huímos de sí mismos si entender que nos miran igual pueblerino, campesino, albañil o "indígena"

Lamentablemente, en el estado de Guerrero, de donde es el autor, se ha manifestado una altísima violencia derivada en asesinatos, desaparición forzada, masacres a estudiantes, como el caso de los 43 normalistas de Ayotzinapa. A pesar de que la ciudad no es el principal motivo de su escritura, sí aparece sugerida en la pérdida de elementos comunitarios y es clave en su proceso creativo. Al respecto, el autor compartió:

Creo que he escrito muy poco acerca de la ciudad o de las ciudades en donde he vivido, mis temáticas siguen siendo las de pueblo, mi comunidad de origen que, por desgracia, ha cambiado mucho por la llegada de los grupos de crimen organizado, la escuela, entre otros. La ciudad en realidad solo me ha servido como punto de comparación en mi proceso creativo. Cuando llegué a vivir por primera vez, me di cuenta de la riqueza que tenemos los que venimos de las comunidades pequeñas porque tenemos la mejor comida, los mejores frutos, los mayores valores humanos como el respeto, entre otras, de ahí es que hago la comparación y escribo acerca de lo que no hay en la ciudad. Otro punto quizá sea, que a partir de que vivo en la ciudad, es cuando más he escrito. . . . No sé si mi trabajo poético sea denunciatorio pero lo que sí puedo afirmar es que, describe una realidad que vivo, lo vive mi pueblo, otros pueblos de México y de Sudamérica e incluso las ciudades principales. El lector es quien al final interpreta el texto. (Entrevista oral) 
Hemos recorrido los espacios planteados por dos escritoras y dos escritores que forman parte del mosaico literario conformado por lenguas, culturas y estéticas, a quienes se les identifica bajo la denominación de literatura indígena; clasificación que debe asumirse con las complejidades que entraña. Tal problematización nos enfrenta a pensar en los prejuicios, así como en las consideraciones erróneas sobre esta literatura. Desde luego, será el lector, interesado o no en estas disquisiciones, quien en la intimidad de su experiencia conformará su verdad con base en su interpretación de la obra literaria. Sin embargo, para los fines del análisis literario que busca horizontes críticos de estudio, conviene observar cómo la etiqueta "indígena" que hemos colocado a la producción literaria escrita en lenguas originarias se corresponde con las temáticas recurrentes, en este caso, del género poético. Desde mi punto de vista, dentro del tipo de literatura híbrida que identifica Lepe Lira, prevalece el tópico de la naturaleza. No es que tal tema sea obligado. La creación literaria supone aventurarse con total libertad creativa hacia experiencias y temáticas, pero resulta imposible apartarse del entorno. En todo caso, esta última afirmación la propongo también como pregunta.

Son valiosos los testimonios y la crítica que ejercen escritores hablantes de alguna lengua indígena a propósito de esta literatura: la escritura, la cultura, el escenario editorial, entre otros temas afines porque, precisamente, permiten al lector mestizo — no estoy segura de emplear "indígena" / "mestizo" como dos condiciones opuestas porque me parece que enteramente no lo son, pero las articularé por ahora - mirar con detenimiento las posturas de escritores indígenas en relación con dicha etiqueta literaria e, incluso, con la palabra indígena. Un ensayo aleccionador o confrontativo es el del escritor tsotsil Mikel Ruiz, titulado "Ni misteriosos ni poéticos" publicado en Tierra Adentro (entre otros). Interpela a Rosario Castellanos —a quien se le ubica en el "Ciclo de Chiapas" y en la literatura indigenista—, en cuanto a la reflexión en torno a ser indígena:

Ser indígena hoy día es no saber lo que realmente es uno. La máscara de lo misterioso y poético, de la que hablaba Castellanos, se fortaleció después del indigenismo. La palabra 
indígena fue la que comenzaron a usar los pueblos para diferenciarse de la sociedad dominante como signo de rebeldía.

¿Es posible saber qué es la literatura indígena si tal denominación como condición de identidad ha sido objeto de múltiples, convergentes y divergentes puntos de vista? Considerando, además, que desde lo hegemónico casi siempre se le ha defnido. La poesía misma responde a esta definición externa sobre lo indígena; el poema de Martín Tonalmeyotl, "Algunos hombres piensan", confronta este hecho al tiempo que presenta una serie de imágenes estereotipadas: "Algunos piensan / que andamos con los pies desnudos / porque estamos aferrados a la tierra ...”. El cierre sintetiza esta postura crítica: "Algunos hombres se toman el derecho / de pensar por nosotros" (51). Ahondar en esto nos llevaría a tomar otro derrotero de análisis, solo propongo observar que, si consideramos esta producción literaria escrita en diversas lenguas indígenas de México de autores nacidos a partir de la segunda mitad del siglo XX, podríamos observar algunas temáticas constantes asumiendo que, favorablemente, surgen nuevas voces, distintas propuestas poéticas que arrancan en varias direcciones.

En términos generales, identifico la presencia de la oralidad y, con esto, destaca la palabra de los mayores, los abuelos y las abuelas cuyos saberes se evocan en los versos. La veta testimonial que puede ser también denunciatoria, puesto que se abordan las condiciones de exclusión, violencia e injusticia que rodean a los pueblos indígenas, la podemos constatar en títulos como Guie'ni zinebe / La flor que se llevó, de Irma Pineda, así como en Tlalkatsajtsilistle / Ritual de los olvidados y el libro que se ha mencionado en este trabajo, ambos de Martín Tonalmeyotl o Tsina rí nàyaxà' / Cicatriz que te mira, de Hubert Matiúwàa. Asimismo, se da respuesta, se problematiza o reflexiona en torno a lo indígena, término que se trata desde diversas ópticas: ¿qué es ser indígena?, ¿cuál es el destino de determinada lengua? Este tópico lo leemos en un rico horizonte de voces, además de las aquí reunidas. Es necesario mencionar que estas preocupaciones no solo se vierten en la poesía, a menudo los escritores se movilizan políticamente para abordar, proponer y construir 
alternativas que impacten favorablemente en las comunidades, así como en diversos ámbitos relacionados con los pueblos indígenas, la lengua y la cultura.

Puede decirse, entonces, que una de las características de esta producción poética es que contiene saberes, preocupaciones, percepciones, sentimientos provenientes desde los espacios más bien reales de sus escritores, en una amalgama, como sucede en la literatura, de elementos reales y ficcionales. Pero me interesa apuntar a los reales, ello no implica en lo absoluto la ausencia de estética o inventiva. En este sentido, los espacios representados en los poemas, sean urbanos o comunitarios, son reflejo de los respectivos ambientes de los escritores. Claramente la ciudad es la referencia del conflicto en los poemas que hemos leído, es el escenario de la aculturación, de la pérdida de los valores cuturales, así como de la adopción de otras dinámicas de vida que, en general, chocan con la propia identidad. Los poemas de las escritoras lo demuestran: en Juana Karen Peñate la metrópoli es un agente que amenaza; en el poema de Mikeas Sánchez, Nueva York aparece con una intrincada carga simbólica vinculada con la migración, la utopía de una mejor calidad de vida, el imperialismo, etcétera.

Hemos visto la relación entre el espacio lírico y el yo poético-autoral, por tal motivo me pareció necesario incorporar los testimonios de los autores con respecto del papel de la ciudad en la escritura. Los poemas sugieren a la ciudad como el espacio no deseado que ha permeado a la comunidad, lo que no impide a los poetas evocar o retomar los elementos más propios de sus respectivos entornos ya sea con una mirada utópica o contrastante. Esta última se percibe en el poema de Wildernain Villegas. La ciudad aparece también como uno de los rostros de la colonización. No obstante, escribir desde dos lenguas, permite posicionar el discurso de resistencia frente a tal hecho. Sin embargo, la mirada crítica no se dirige exclusivamente a la ciudad, también a la comunidad, especialmente en la poesía de Martín Tonalmeyotl: "donde a los niños se les enseña a pisotear su idioma / donde nuestros hermanos han desenterrado su ombligo / para no sentir el sudor campesino" (27). 
Se alza la voz a propósito de la perpetua colonización de los pueblos indígenas y hacia otras agudas problemáticas sociales. En este tenor, Lepe Lira aborda la literatura como un acto de resistencia:

.. la colonialidad también produce decolonialidad y los textos de los escritores indígenas nos muestran un lugar de creación y resistencia que sacude al sistema mundo y decoloniza los relatos de la diferencia, pues genera una literatura en dos lenguas: las lenguas indígenas y el español, que traspasa dos o más mundos. ${ }^{8}$ (18)

A manera de conclusión podría decirse que la comunidad, referida constantemente por los poetas como espacio de creación (en sus testimonios) y/o tema de escritura (en la poesía), es aquella que, dentro de ciertos límites geográficos, conforma un escenario de dinámicas cotidianas específicas, el empleo de una o más lenguas, saberes, manifestaciones culturales y religiosas, como las danzas en Chapultenango que se efectúan el 2 de febrero, por poner un ejemplo. Lo que subrayo es la cuestión de pertenencia de parte de los poetas hacia la comunidad; si revisamos cada libro de poesía —el título Corazón de selva de Juana Karen, alude claramente a la impor-

${ }^{8} \mathrm{La}$ cita forma parte de la hipótesis de la autora en relación con aquello que ha sostenido el imaginario colonial sobre los pueblos indígenas. Hace la aclaración que, si bien el colonialismo como momento histórico pertenece al pasado, en los países latinoamericanos se deja ver la huella de la diferencia colonial que se configuró hace más de quinientos años, misma que permanece en las políticas públicas de estos países; asimismo, siguiendo a Pablo González Casanova, se ha dado un colonialismo interno que ha propiciado una mirada peyorativa hacia los elementos prehispánicos de las expresiones políticas y estéticas de los pueblos, de acuerdo con la autora. Y, en síntesis, el imaginario colonial se sostiene, según Lepe Lira, por relatos de la diferencia colonial: "Los relatos raciales (sobre el color de la piel), geopolíticos (sobre la cartografía y distribución de los recursos) y del conocimiento (sobre los saberes y la subjetividad) sostienen una matriz de poder que alimenta el sistema mundo con su economía capitalista, volviendo natural la colonialidad y las condiciones de vida de los pueblos latinoamericanos" (Lepe, Relatos de la diferencia 18). 
tancia del lugar de origen- encontraremos la presencia constante de la comunidad, de la vida comunitaria: a veces se representa desde una nostalgia derivada de la experiencia de su autor; otras, mediante el tono testimonial que denuncia la marginación, exclusión, violencia, provenientes no solo del exterior, sino que se dan, también, en las propias comunidades, derivadas de problemáticas sociales complejas; de igual manera, se coloca atención al ámbito religioso y espiritual. Añado el hecho de que en la poesía que hemos revisado se plantea, quizá sin intención, una representación binaria de ciudad / comunidad-naturaleza, sobre todo en los poemas de Juana Karen Peñate y Wildernain Villegas. Lo anterior mencionado son algunas manifestaciones de dichos tópicos, por supuesto no son todos, pero me parece que son visibles.

Finalmente, en la poesía de los cuatro escritores, principalmente en la de Martín Tonalmeyotl, se bifurcan los espacios comunitarios con los urbanos. En cuanto a la relación del autor con el texto, es evidente el tema del desplazamiento; cada uno compartió su testimonio en relación con migrar a otros entornos. Este dato biográfico no necesariamente se refleja en el poema, pero la experiencia del desplazamiento está latente en los libros de poesía. En el caso del poema de Mikeas Sánchez, la voz lírica permite el desplazamiento de la comunidad hacia Nueva York: lanza el deseo de que Nasakobajk acompañe a la ore'yomo migrante. Me parece que las migraciones de los escritores, cortas o extensas, han sido fuente de escritura e interés por el espacio urbano con una mirada, casi siempre, desde la comunidad; los autores aquí citados expresan una clara referencia a ésta: es el espacio, aunque no exclusivo, de la lengua materna, pero me asalta una pregunta en tanto es una posibilidad: ¿cuáles son las preocupaciones literarias de los escritores indígenas que nacieron en la ciudad? Por último, otra veta posible de abordaje en relación con el tema visto es cómo se ha modificado la presencia de la ciudad en el indigenismo literario en comparación con la literatura indígena actual. 


\section{Bibliografía}

Aguilar, Yásnaya y Jan Terje. La lengua zoque. Centro de Investigaciones Multidisciplinarias sobre Chiapas y la Frontera Sur / Universidad Nacional Autónoma de México, 2017.

Alonso Bolaños, Marina. "Los zoques bajo el volcán. Microhistorias de la erupción de El Chichonal, Chiapas”. 2011. El Colegio de México, Tesis doctoral, dirigida por Juan Pedro Viqueira.

Beristáin, Helena. Análisis e interpretación del poema lírico. Universidad Nacional Autónoma de México, 1989.

Blanco, José. La literatura en la Nueva España. Cal y Arena, 1989.

De la Garza, Mercedes. El legado escrito de los mayas. Fondo de Cultura Económica, 2012.

Editorial Herder México. “Juana Karen”. herder.com.mx/es/libros-books/corazon-de-selva-ipusikal-matyelum/juana-karen/ pluralia.

García Canclini, Néstor. "México 2010: una ciudad que improvisa su globalización”. Alteridades, no. 26, 2003, pp. 7-14.

León-Portilla, Miguel. Mitos prehispánicos. Universidad Autónoma de México, 2016.

- Quince poetas del mundo nábuatl. Diana, 1994.

Lepe, Luz María. Lluvia y viento, puentes de sonido. Literatura indigena y crítica literaria. Universidad Autónoma de Nuevo León, 2010.

- Relatos de la diferencia y literatura indígena. Travesías por el sistema mundo. Grañén / Porrúa / Universidad Nacional Autónoma de México / Universidad Autónoma de Querétaro, 2018.

López Austin, Alfredo y Leonardo López Luján. El pasado indigena. Fondo de Cultura Económica, 2011.

Peñate, Juana Karen. Corazón de selva / Ipusik'al matye'lum. Pluralia, 2013.

. Entrevista oral. Por Krishna Naranjo Zavala, 03 junio 2019.

Pulido Tirado, Genara. "El canon literario en América Latina”. Signa: Revista de la Asociación Española de Semiótica, no. 18, pp. 99-114. Ruiz, Mikel. "Mu yanuk mu nichimal jbakutik / Ni misteriosos ni poéticos". Tierra Adentro, 21 febrero 2019, www.tierraadentro. cultura.gob.mx/ni-misteriosos-ni-poeticos/ 
Sánchez, Mikeas. Kobikiajubäjjaye / Selección poética. Conaculta, 2012. Tonalmeyotl, Martín. Entrevista oral. Por Krishna Naranjo Zavala, 03 junio 2019.

Tonalmeyotl, Martín, coordinador. "Xochitlájtoli: Juana Karen”. Círculo de poesía. Revista electrónica de literatura circulodepoesia. com/2017/11/xochitlajtoli-juana-karen/

. "Xochitlájtoli: Wildernain Villegas Carrillo". Círculo de poesía. Revista electrónica de literatura, circulodepoesia.com/2019/01/xochitlajtoli-wildernain-villegas-carrillo/

Villegas, Wildernain. Entrevista oral. Por Krishna Naranjo Zavala, 10 de febrero de 2021.

- Lluvia que la noche dicta / Áak'abe' ku ya'alik táan u k'áaxal ja'. Secretaría de la Cultura y de las Artes de Yucatán, 2012. 